\title{
Integrated Design of a Planar Maglev System for Micro Positioning
}

\author{
Mei-Yung Chen ${ }^{1}$, Chia-Feng Tsai ${ }^{2}$, Hsuan-Han Huang ${ }^{2}$ and Li-Chen Fu ${ }^{2}$ \\ 1.Department of Electrical Engineering, China Institute of Technology, \\ Taipei, Taiwan, R.O.C.(mychen@cc.chit.edu.tw) \\ 2. Department of Electrical Engineering, National Taiwan University,
}

Taipei, Taiwan,R.O.C.(lichen@ccms.ntu.edu.tw)

\begin{abstract}
According to what the 6-DOF magnetic levitation system has proposed at last year. In the continuous work, this research desire to upgrade positioning precision. The redesign concept attempts to keep the good performance in the whole journey of moving rather than the point-to-point positioning precision. Based on these concepts, the force model was modified in considering the variation from the displacement to the magnetic forces first, and avoids the constraint of the attractive levitation in replacing the repulsive levitation. Finally, the concept of relative place to build the measuring system was adopted. All of the performance of the improved framework will be demonstrated in the experimental results.
\end{abstract}

Keywords: Maglev, Precision positioning, Adaptive sliding-mode controller

\section{Introduction}

Electrical machines have existed since electromagnetic phenomena were first systematically researched in the $19^{\text {th }}$ Century. Traditionally, the electromagnetic drives are rotational motors, and the rotational movement is converted by means of the transmissions when the linear movement is required for application. Mostly, the piezoelectric actuators only handle the small moving range[1][2], the ball-screws cause disturbances and backlash due to roughness of the bearing elements[3][4], and the linear motors have end effect in a motion stroke. Therefore, other proper resolutions was needed that can extend their application fields and satisfy these requirements as well-no contamination, no friction, high speed, and can be operated in a high vacuum environment and also a clean room. Hereto magnetic levitation (abbreviated as Maglev) mechanism was gener-

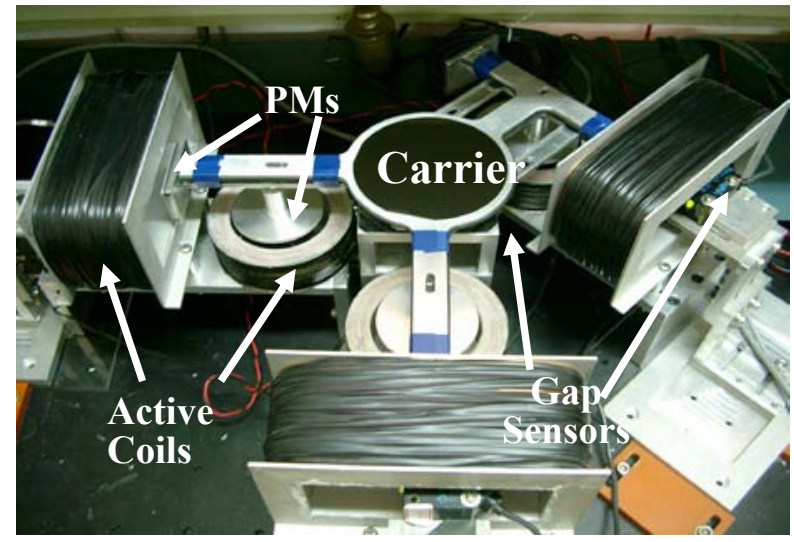

Figure 1. Photograph of the improved framework

ally believed that it is the optimal solutions[5][6].

Based on the design theories and experience of the planar Maglev system, which was proposed at last year[7], in development, the carrier in adding a one-shaped structure was redesign and replacing the cylindrical coils with larger one to generate the uniform magnetic field near the surface of the coils. Likewise, the relative circular magnets are replaced with larger one to decrease the air gap and generate the powerful magnetic forces, too. Additionally, in order to have the expectable larger moving range $(\mathrm{mm})$, the repulsive levitation instead of the attractive one was employed. The photograph of the improved framework is shown in Fig. 1.

\section{Magnetic Forces and System Modeling}

\subsection{Magnetic forces formulation}

Based on the previous research[7][8], from the theoretical analysis, the magnetic forces with the multiplication of displacement function and fed current can be presented and simplified as: 


$$
F_{r e c / c y l, \perp}=g_{\text {rec/cyll, }}(x, y, z) u_{r e c / c y l},
$$

where $u$ is the fed current, subscript “ $\perp$ " is denoted as the forces act perpendicular to the surface of the permanent magnet, and rec and cyl are denoted as the rectangular and cylindrical coil, respectively.

Actually, the height in levitation is always stationary in this system. Therefore, for the bottom subsystem, what most concern will be the influence of variation of the displacement on the magnetic forces in the horizontal direction rather than in the vertical direction. When a Taylor series expansion of the analytical magnetic force equation is truncated after the first order term, within a small range around the nominal position, the forces formulation Eq.(1) can be expressed as:

$$
\begin{aligned}
\delta F(d, u) & =\left.\delta F_{0}\right|_{p=p_{0}, u=u_{0}}+\left.\frac{\partial F}{\partial d}\right|_{d=d_{0}, u=u_{0}} \cdot \delta p+\left.\frac{\partial F}{\partial u}\right|_{d=d_{0}} \cdot \delta u \\
& =\delta F_{0}+k_{d} \cdot \delta d+k_{u} \cdot \delta u
\end{aligned}
$$

where $k_{d}$ and $k_{u}$ represents the force-displacement factor and force-current factor respectively. Neglecting the hysteresis, $\delta F_{0}$ is zero, and removing ' $\delta$ ' in the final form, the force equation with a linear expression becomes

$$
\begin{aligned}
& F_{c y l}(d, u)=k_{d, c y l} \cdot d_{o, c y l}+k_{u, c y l} \cdot u_{c y l}, \\
& F_{r e c}(d, u)=k_{d, r e c} \cdot d_{o, r e c}+k_{u, r e c} \cdot u_{r e c},
\end{aligned}
$$

where the $d_{o, c y l}$ means the absolute distance form the measured point to the center of the surface of cylindrical coil in the horizontal direction and the $d_{o, \text { rec }}$ means the distance form the measured point to the center of the surface of rectangular coil in the direction perpendicular to this surface.

Finally, there is a fairly important point that needs to be considered. As mentioned in the force model simplification, we just use the half-part curve of the full-range distribution for representation in virtue of the symmetry. Therefore, to represent the full-range distribution well in the linearized form, there should be some modifications. According to the symmetry of the force distribution, we can apply the distribution behavior on one side to describe that on the other side and hence Eq.(3) has to be rewritten as follows:

$$
\begin{aligned}
& F_{c y l}(d, u)=k_{d, c y l} \cdot\left|d_{o, c y l}\right|+k_{u, c y l} \cdot u_{c y l}, \\
& F_{r e c}(d, u)=k_{d, r e c} \cdot\left|d_{o, r e c}\right|+k_{u, r e c} \cdot u_{r e c} .
\end{aligned}
$$

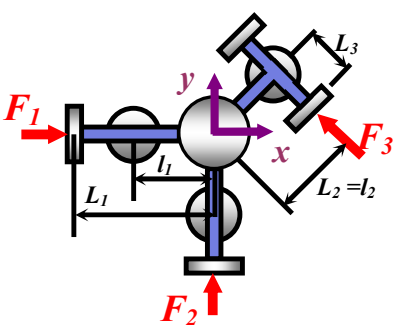

(a)

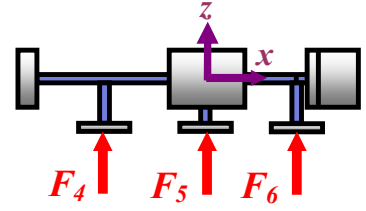

(b)
Figure 2. Carrier structure and force relation. (a) Top view of the carrier. (b) Front view of the carrier

\subsection{System modeling}

The component of the planar Maglev system include: six PMs attached on the moving part so-called carrier, while six coils mounted on a fixed part. Three of these six coils are arranged in the bottom and mainly generate levitating forces as well as torques around $x$ - and $y$-axis. And the three side-coils with rectangle shaped mainly produce lateral forces and torque around $z$-axis as well. Figure 2 shows the design concepts combining with the forces relations.

Then, some assumptions are made. First, it is assumed that the mutual effects in this system are very small and can be ignored. The second, in our operations, the performance of carrier's translation is mainly concerned and its rotation is just regulated. That means the angular displacement of the carrier around each axis is very small. Due to these small angular displacements, it is assumed that the attitude around $z$-axis will not be coupled with that around the other two axes. Due to the system's structure, by the Newton's and Euler's law, the total forces and torques equations can be expressed as:

$$
\begin{aligned}
& \sum F_{x}=F_{1}-\sqrt{2} / 2 F_{3}=m \ddot{x}, \\
& \sum F_{y}=F_{2}+\sqrt{2} / 2 F_{3}=m \ddot{y}, \\
& \sum F_{z}=F_{4}+F_{5}+F_{6}-m g=m \ddot{z}, \\
& \sum T_{x}=-F_{5} l_{1}+\sqrt{2} / 2 F_{6} l_{2} \cong I_{x x} \ddot{\varphi}, \\
& \sum T_{y}=F_{4} l_{1}-\sqrt{2} / 2 F_{6} l_{2} \cong I_{y y} \ddot{\phi}, \\
& \sum T_{z}=F_{3} L_{2} \cong I_{z z} \ddot{\theta},
\end{aligned}
$$

where $m=0.625 \mathrm{~kg}, l_{l}=0.16 \mathrm{~m}, L_{l}=0.1 \mathrm{~m}, l_{2}=L_{2}=0.12 \mathrm{~m}$, and $l_{3}=0.06 \mathrm{~m}$. From Eq.(5), the governing equations of the rigid body can be expressed in the vector form, and after substituting the position function with the linearized result from Eq.(5), the motion equation can be express as: 


$$
M \ddot{X}=\bar{K}_{u} U+\bar{K}_{d} D_{o}-G,
$$

each variable is defined as follows:

$$
\begin{aligned}
& X=\left[\begin{array}{llllll}
x & y & z & \varphi & \phi & \theta
\end{array}\right]^{T}, \\
& M=\operatorname{diag}\left[m, m, m, I_{x x}, I_{y y}, I_{z z}\right] \text {, } \\
& U=\left[\begin{array}{llllll}
u_{1} & u_{2} & u_{3} & u_{4} & u_{5} & u_{6}
\end{array}\right]^{T}, \\
& G=\left[\begin{array}{llllll}
0 & 0 & m g & 0 & 0 & 0
\end{array}\right]^{T} \text {, } \\
& D_{o}=\left[\left|d_{o, r e c, x}\right|\left|d_{o, r e c, y}\right|\left|d_{o, r e c, T}\right|\left|d_{o, c y l, x}\right|\left|d_{o, c y l, y}\right|\left|d_{o, c y l, T}\right|\right]^{T}, \\
& \bar{K}_{d}=C \cdot \operatorname{diag}\left[k_{d, r e c, x}, k_{d, r e c, y}, k_{d, r e c, T}, k_{d, c y l, x}, k_{d, c y l, y}, k_{d, c y l, T}\right] \text {, } \\
& \bar{K}_{u}=C \cdot \operatorname{diag}\left[k_{u, r e c, x}, k_{u, r e c, y}, k_{u, r e c, T}, k_{u, c y l, x}, k_{u, c y l, y}, k_{u, c y l, T}\right] \text {, and }
\end{aligned}
$$$$
C=\left[\begin{array}{cccccc}
1 & 0 & -\sqrt{2} / 2 & 0 & 0 & 0 \\
0 & 1 & \sqrt{2} / 2 & 0 & 0 & 0 \\
0 & 0 & 0 & 1 & 1 & 1 \\
0 & 0 & 0 & 0 & -l_{1} & \sqrt{2} / 2 l_{2} \\
0 & 0 & 0 & l_{1} & 0 & -\sqrt{2} / 2 l_{2} \\
0 & 0 & L_{2} & 0 & 0 & 0
\end{array}\right],
$$

where the subscript $d / u$ denotes the force-displacement or force-current factor, rec/cyl denotes the rectangular or cylindrical coil, and the final one $x / y / T$ denotes the location of the subsystem in the $x, y$ direction or T-shaped bearing.

In this dynamic model, in addition to the dimensions of the configuration and the distribution and strength of the magnetic force can be measured in advance, so the system's six states: $x, y, z, \varphi, \phi$, and $\theta$ can be on-line measured.

\section{Controller Design}

Refer to the linearized plant model Eq.(6) with an additional uncertainty term:

$$
M \ddot{X}=\bar{K}_{u} U+\bar{K}_{d} D_{o}-G+w,
$$

where the additional term $w$ is a $6 \times 1$ vector considered as the noise and input disturbances due to imperfect system model and states transformation, and it is assumed its valves are bounded. According to Eq.(7), the bias command input is decided with

$$
U_{\text {Bias }}=\bar{K}_{u}^{-1} G \text {. }
$$

If the control input was designed as the sum of one specific controller $U_{c t r l}$ and the bias $U_{\text {Bias }}$ as:

$$
U=U_{c t r l}+U_{\text {Bias }},
$$

then Eq.(7) cab be rewrote as

$$
M \ddot{X}=\bar{K}_{u} U_{c t r l}+\bar{K}_{d} D_{o}+w .
$$

\subsection{Controller Design}

Before the design of adaptive controller, the error state $E$ was defined as the difference between current state $X$ and reference signal $X_{f}$, i.e. $E=X-X_{f}$. Therefore, Eq.(10) can be rewritten as:

$$
\ddot{E}=-\ddot{X}_{r}+A_{u} U_{c t r l}+B_{d} D_{o}+W,
$$

where $A_{u}=M^{-1} \bar{K}_{u}, B_{d}=M^{-1} \bar{K}_{d}$, and $W=M^{-1} w$. Furthermore, divide the last term $W$ into a constant uncertainty $W_{\text {const }}$ and a varying uncertainty $W_{\text {var }}$, and represent the error-state equation with

$$
\ddot{E}=-\ddot{X}_{r}+A_{u} U_{c t r l}+B_{d} D_{o}+W_{c o n s t}+W_{v a r} .
$$

Due to the assumption of the additional term $w$, it leads the varying uncertainty to be bounded, $\left\|W_{\text {var }}\right\| \leq W_{M A X}$.

Next, design a sliding surface $S$ as follow:

$$
S=\dot{E}+\Lambda E,
$$

where $\Lambda=\operatorname{diag}\left[\lambda_{1}, \lambda_{2}, \lambda_{3}, \lambda_{4}, \lambda_{5}, \lambda_{6}\right]^{T}$, and $\forall \lambda_{i}>0$.

In this application, the state error $E$ and the derivative of $E$ will be regulated to zero, simultaneously. Due to this reason, if the sliding surface tends to zero within finite time can be proved, then $E$ and $\dot{E}$ are also forced to zero exponentially. To relate the sliding surface to the dynamics of motion, the time derivative of the sliding surface is:

$$
\dot{S}=\ddot{E}+\Lambda \dot{E} .
$$

In this research, an adaptive controller is applied in this research, which is capable of estimating parameters of the system online while controlling the system simultaneously. After the system parameters have estimated, Eq.(12) with these estimates in the control command can be used. So, the estimates acquired from the online estimator can be substituted and derive as:

$$
U_{c t r l}=\hat{A}_{u}^{-1}\left(v-\hat{B}_{d} D_{o}-\hat{W}_{\text {const }}+\ddot{X}_{r}-N \cdot \operatorname{sat}(S)\right),
$$

where $N$ is a positive value and $\operatorname{sat}($.$) is the saturation func-$ 
tion defined as:

$$
\operatorname{sat}(S) \equiv\left[\operatorname{sat}\left(s_{1}\right), \operatorname{sat}\left(s_{2}\right), \operatorname{sat}\left(s_{3}\right), \operatorname{sat}\left(s_{4}\right), \operatorname{sat}\left(s_{5}\right), \operatorname{sat}\left(s_{6}\right)\right]^{T},(16
$$

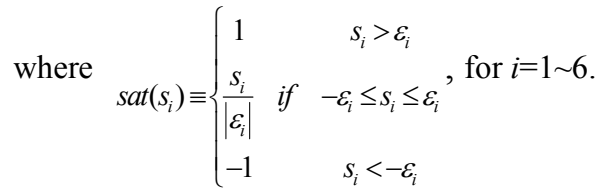

Therefore, in Eq.(15), the symbols was used with head to denote the estimation terms of their real values, and afterward, applying the adaptive law to design the estimation terms. Additionally, this controller was modified in the designing boundary value of the saturation function to satisfy $\|N\| \geq W_{\text {MAX }} \geq\left\|W_{\text {var }}\right\|$. Then, the adding PID controller, $v$, can be expressed as

$$
\begin{aligned}
& v=-G(t), \\
& G(t)=K_{P} \cdot E+K_{I} \cdot \int E+K_{D} \cdot \frac{d E}{d t},
\end{aligned}
$$

where $K_{D}, K_{P}$, and $K_{I}$, are parameters to be tuned. Subsequently, from designing the realizable liner controller $G(t)$, which can merge $\Lambda \dot{E}$ term into the controller. Thus, substituting Eq.(15) into Eq.(14), we can obtain:

$$
\dot{S}=\tilde{A}_{u} U_{C t r l}+v+\tilde{B}_{d} D_{o}+\tilde{W}_{\text {const }}-N \cdot \operatorname{sat}(S),
$$

where $\tilde{A}_{u}, \tilde{B}_{d}$, and $\tilde{W}_{\text {const }}$ are denoted as the estimation errors with $\tilde{A}_{u}=A_{u}-\hat{A}_{u}, \quad \tilde{B}_{d}=B_{d}-\hat{B}_{d}, \quad$ and $\tilde{D}_{\text {const }}=D_{\text {const }}-\hat{D}_{\text {const }}$, respectively. If the estimation errors can converge to zero asymptotically, the $\dot{S}$ will become

$$
\dot{S}=v-N \cdot \operatorname{sat}(S)
$$

According to the theory of sliding mode control[9], Eq.(19) reveals that time derivative of the sliding surface $\dot{S}$ will decrease when $S$ is positive and will increase when $S$ is negative until the sliding surface converges to zero.

\subsection{Stability Analysis}

As mentioned above, in order to eliminate the estimation errors, the adaptive laws have to be found out. Applying direct Lyapunov method and defining a Lyapunov function candidate $V$, it is a positive definite function:

$$
\begin{aligned}
V= & \frac{1}{2} S^{T} S+\frac{1}{2} \operatorname{tr}\left(\tilde{A}_{u}^{T} \Phi_{1}^{-1} \tilde{A}_{u}\right)+\frac{1}{2} \operatorname{tr}\left(\tilde{B}_{d}^{T} \Phi_{2}^{-1} \tilde{B}_{d}\right)+ \\
& \frac{1}{2} \operatorname{tr}\left(\tilde{W}_{\text {const }}^{T} \Phi_{3}^{-1} \tilde{W}_{\text {const }}\right)
\end{aligned},
$$

where $\Phi_{1}^{-1}, \Phi_{2}^{-1}$, and $\Phi_{3}^{-1}$ are all positive diagonal matrixes. Then the differential of the Lyapunov function candidate with time variance can be express as:

$$
\begin{aligned}
\dot{V}= & S^{T} \dot{S}+\operatorname{tr}\left(\tilde{A}_{u}^{T} \Phi_{1}^{-1} \dot{\tilde{A}}_{u}\right)+\operatorname{tr}\left(\tilde{B}_{d}^{T} \Phi_{2}^{-1} \dot{\tilde{B}}_{d}\right)+ \\
& \operatorname{tr}\left(\tilde{W}_{\text {const }}^{T} \Phi_{3}^{-1} \dot{\tilde{W}}_{\text {const }}\right)
\end{aligned}
$$

Substitute Eq.(19) into Eq.(21). Then, it can be rearranged as following:

$$
\begin{aligned}
\dot{V} & =S^{T}(v-N \cdot \operatorname{sat}(S))+\operatorname{tr}\left(\tilde{A}_{u}^{T}\left(S U_{C t r l}^{T}+\Phi_{1}^{-1} \dot{\tilde{A}}_{u}\right)\right) \\
& +\operatorname{tr}\left(\tilde{B}_{d}^{T}\left(S D_{o}^{T}+\Phi_{2}{ }^{-1} \dot{\tilde{B}}_{d}\right)\right)+\operatorname{tr}\left(\tilde{W}_{\text {const }}^{T}\left(S+\Phi_{3}{ }^{-1} \dot{\tilde{W}}_{\text {const }}\right)\right)
\end{aligned}
$$

Now, adaptive laws were derived as:

$$
\begin{gathered}
\dot{\tilde{A}}_{u}=-\dot{\hat{A}}_{u}=-\Phi_{1} S U_{C t r l}^{T}, \\
\dot{\tilde{B}}_{d}=-\dot{\hat{B}}_{d}=-\Phi_{2} S D_{o}^{T}, \\
\dot{\tilde{W}}_{\text {const }}=-\dot{\hat{W}}_{\text {const }}=-\Phi_{3} S .
\end{gathered}
$$

In order to maintain stability and to prevent saturation of the control effort, $\hat{A}_{u}$ must be bounded away from zero in Eq.(23). One method to avoid $\hat{A}_{u}$ going through zero is to modify the adaptive law using a projection method. Such a modification does not affect the convergence of $\tilde{A}_{u}$ and is achieved by using a prior knowledge of $A_{u}$, such that $A_{u} \geq A_{u}\left(t_{0}\right)$, where $A_{u}\left(t_{0}\right)$ is defined as the lower bound. Applying the projection method to the adaptive law, we obtain

$$
\dot{\hat{A}}_{u}(t)=\left\{\begin{array}{lcc}
\Phi_{1} S U_{A S}^{T} & \text { if } \quad \hat{A}_{u}(t) \geq A_{u}\left(t_{0}\right) \\
0 & \text { otherwise }
\end{array} .\right.
$$

At the initial time, $\hat{A}_{u}\left(t_{0}\right)$ is chosen so that $\hat{A}_{u}\left(t_{0}\right)>A_{u}\left(t_{0}\right)$. If these equations hold, Eq.(28) will become

$$
\dot{V}=S^{T}(v-N \cdot \operatorname{sat}(S))=S^{T} \dot{S} \leq 0 .
$$

By using the Barbalat's Lemma[9], we can establish that $S \in L_{2}, \quad \dot{S} \in L_{\infty}$ and so that: $|S(t)| \leq \varepsilon$. Due to $S$ convergence to zero, it can be readily verified that $E$ coverage to zero asymptotically. In other words, the all state variables and time derivatives all converge to zero eventually, which is the goal of designing the controller for this system.

\section{Experimental Results}


The experimental hardware, including the main body, sensor system, driver system and controller hardware have built. A number of experimental results, including the transient and the steady-state responses in different situations, will also be provided in this section to demonstrate the performance of this system with the controller presented in section 3 .

To test the largest moving range, $\mathrm{mm}$-scale motion, we try to execute the positioning motion in $y$ direction and regulate the other five states to zero position. Figure 3 shows the transient responses to the situation with the largest initial transitional displacement range, i.e., $7 \mathrm{~mm}$ in this system. From the trajectories of state, one can see that the all error signals converge to the set-points.

In addition to the $\mu m$-scale motion, the system response for a continuing stepping command with each step equal to $0.1 \mathrm{~mm}$ are shown in Fig 4 . The step-train test result demonstrates the satisfactory performance in $y$-axis motion and stiffness in other 5 axes. The final precision is made up $5 \mu \mathrm{m}$ in translation which is estimated to reach a limit of the sensor device. To explain the meaning of the result, it is necessary to consider the resolving capability of the measuring device firstly. Namely, the repeatable accuracy is about $5 \mu \mathrm{m}$.

Additionally, the other way to show the tracking capability is to profile a desired circle. Figure 5 present the experimental results of this circling motion which radius is equal to $2.0 \mathrm{~mm}$ at frequency $0.4 \mathrm{~Hz}$.

According to what we have revealed in above experimental results, here the positioning precision will be compared with previous work. From Table. 1, it is evident to see that the positioning precision of the improved configuration is better than that before.

\section{Conclusion}

As a continuous work, in this paper, the upgraded positioning precision has successfully improved through some redesign concepts. These redesign on the configuration including the adoption of repulsive levitation, increase of the actuating forces, and consolidation of the structure of carrier. By experimental results, excellent performance indices have been observed which ascertain that the objectives were successfully achieved; the upgrade of the whole performance when moving, precision positioning, and expectable larger moving range.
Table 1: The root-mean-square-error (RMSE) performance of the previous work and of current work

\begin{tabular}{|c|c|c|c||}
\hline \hline RMSE & $\begin{array}{c}\text { Largest } \\
\text { motion }\end{array}$ & $\begin{array}{c}\text { Step-train } \\
\text { response }\end{array}$ & $\begin{array}{c}\text { Circle } \\
\text { motion }\end{array}$ \\
\hline Previous work & $0.8 \times 10^{-3} \mathrm{~mm}$ & $10 \times 10^{-3} \mathrm{~mm}$ & $0.1 \mathrm{~mm}$ \\
\hline Current work & $0.5 \times 10^{-3} \mathrm{~mm}$ & $0.5 \times 10^{-3} \mathrm{~mm}$ & $4 \times 10^{-3} \mathrm{~mm}$ \\
\hline
\end{tabular}

\section{Acknowledgement}

This research is sponsored by National Science Council, R.O.C., under the grant NSC-93-2752-E-002-007-PAE.

\section{Bibliography}

[1] Sitti, M. and Hashimoto, H., "Two-dimensional fine particle positioning using a piezoresistive cantilever as a micro/nano-manipulator", 1999 IEEE International Conference on Robotics and Automation, Vol. 4, pp. $2729-2735,1999$.

[2] Hector M. Gutierrez, Paul I. Ro. "Sliding-Mode Control of a Nonlinear-Input System: Application to a Magnetically Levitated Fast-Tool Servo", IEEE Tran. on Industrial Electronics ,Vol. 45, no. 6, pp. 921 -927, 1998.

[3] Li Xu and Bin Yao, “Adaptive robust precision motion control of linear motors with negligible electrical dynamics: theory and experiments", IEEE/ASME Tran. on Mechatronics, Vol. 6 Issue: 4, pp. 444 -452, 2001.

[4] Pai-Yi Huang, Yung-Yew Chen and Min-Shin Chen "Position-dependent friction compensation for ballscrew tables", Proceedings of the 1998 IEEE International Conference on Control Applications, Vol. 2, pp. 863-867, 1998.

[5] Kwang Suk Jung and Yoon Su Baek, "Study on a novel contact-free planar system using direct drive DC coils and permanent magnets," IEEE/ASME Trans. Mechatron., vol. 7, no. 2, March 2002.

[6] Lex Molenaar, "A novel planar magnetic bearing and motor configuration applied in a positioning stage," Ph.D thesis, Delft University of Technology, 2000.

[7] Mei-Yung Chen, Tzuo-Bo Lin, Chia-Feng Tsai, and Li-Chen Fu, "Novel Design of a 6-DOF Planar Maglev Positioning System," IEEE/ASME International Conference on Advanced Intelligent Mechatronics, pp,.290-295, 2003. 

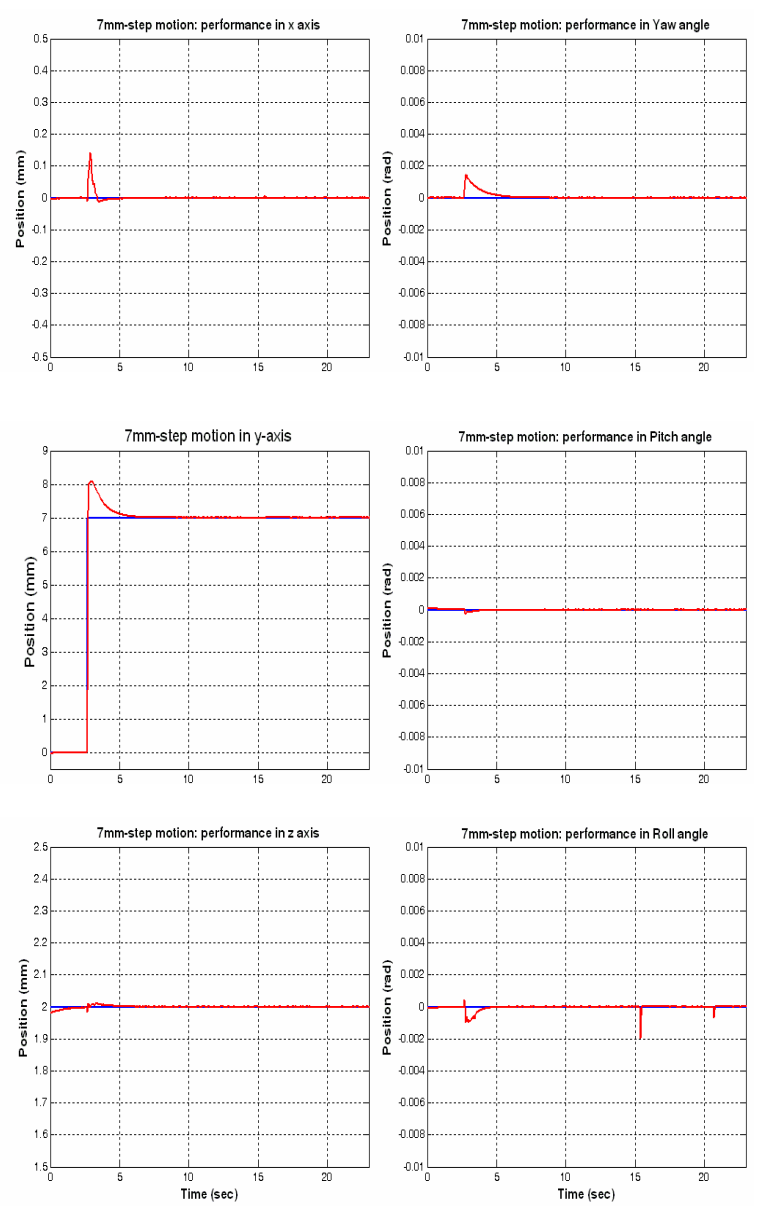

Figure 3: Transient responses of the carrier with largest translation displacement.

[8] J. G. David, Introduction to Electrodynamics. Prentice-Hall, Inc., Englewood Cliffs, New Jersey, 1981.

[9] Jean-Jacques E. Slotine, Weiping Li., Applied nonlinear control, Prentice Hall, 1990.
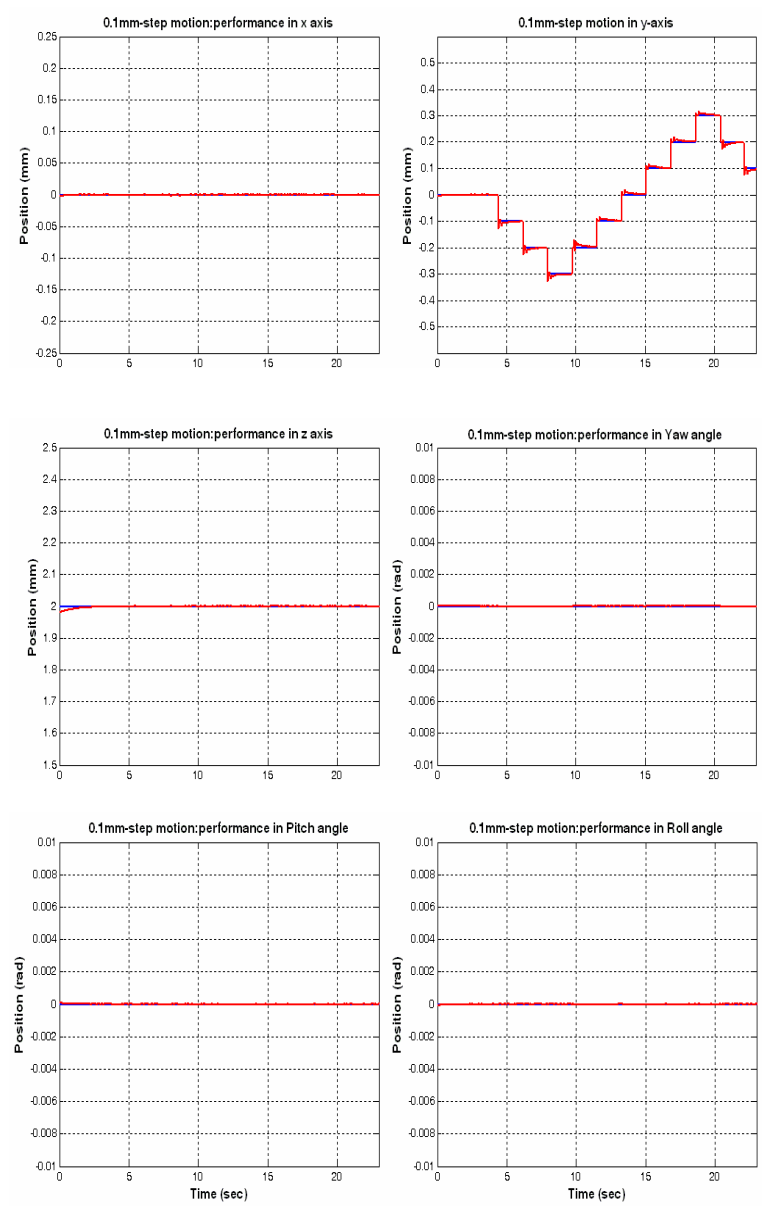

Figure 4: 0.1mm step input control.

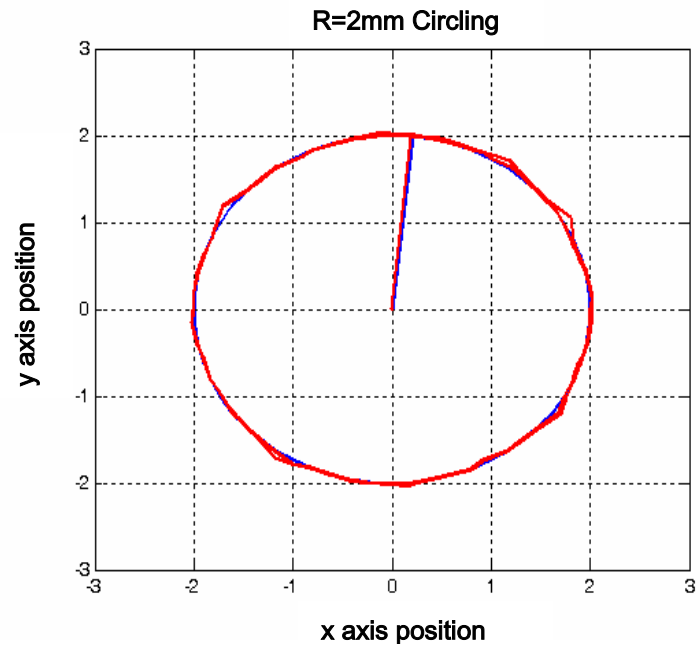

Figure 5: 2.0mm, $0.4 \mathrm{~Hz}$ circling motion in XY plot. 